\title{
Model for Predicting Phase Transformation and Yield Strength of Vanadium Microalloyed Carbon Steels
}

\author{
Ho-Hung KUO, ${ }^{1,4)}$ Minoru UMEMOTO, ${ }^{1,4)}$ Kazuki SUGITA, ${ }^{2)}$ Goro MIYAMOTO ${ }^{3,4)}$ and Tadashi FURUHARA ${ }^{3,4)}$ \\ 1) Department of Production Systems Engineering, Toyohashi University of Technology, Toyohashi, Aichi, 441-8580 Japan. E-mail: \\ kuo@martens.me.tut.ac.jp, umemoto@martens.me.tut.ac.jp 2 2) Department of Materials Science and Engineering, \\ Kyoto University Graduate School of Engineering, Kyoto, 606-8501 Japan. E-mail: sugita.kazuki.3v@kyoto-u.ac.jp \\ 3) Institute for Materials Research, Tohoku University, Sendai, Miyagi, 980-8577 Japan. E-mail: miyamoto@imr.tohoku.ac.jp, \\ furuhara@imr.tohoku.ac.jp $\quad 4)$ "Fundamental Studies on Technologies for Steel Materials with Enhanced Strength and \\ Functions" Consortium of JRCM (The Japan Research and Development Center for Metals).
}

(Received on October 17, 2011; accepted on February 8, 2012)

\begin{abstract}
An integrated model for predicting recrystallization, phase transformation and yield strength of vanadium-microalloyed carbon steel (V-steel) is developed. Two effects of vanadium addition on recrystallization are assumed: one is the solute-drag effect on mobility of grain boundary, the other is pinning-effect on austenite grain growth due to vanadium carbide (VC) precipitate in austenite. The austenite grain size is considered as the control variable for nucleation density in grain corner, grain boundary, and grain interior during phase transformation. Thermodynamic data for transformation including para-equilibrium of carbon concentration and driving force were calculated using ThermoCalc software. The vanadium addition leads to $\alpha / \gamma$-interphase VC precipitation in ferrite, which accelerates the diffusion rate of carbon in austenite at $\alpha / \gamma$ interface and increases nucleation sites for intragranular ferrite transformation. In consequence, the ferrite fraction and grain size are increased. Brandt model ${ }^{27)}$ is conducted to predict pearlite transformation. The lamellar spacing is considered as a function of carbon concentration and undercooling. The alloying elements, ferrite and pearlite fractions, ferrite grain size, and lamellar spacing were taken into account for predicting strength of $\mathrm{V}$-free steels. Modified Ashby-Orowan equation is then used to calculate the VC precipitation strengthening of V-steels. Using this model the calculated results obtained are in good agreement with experimental results.
\end{abstract}

KEY WORDS: vanadium; carbon steel; recrystallization; phase transformation; yield strength.

\section{Introduction}

Thermo-mechanical processing has been developed to improve mechanical properties of forging vanadiummicroalloyed medium carbon steels (V-steels) for automotive components. This process consists of controlled forging and isothermal or continuous cooling treatments.

Controlled forging provides a deformation to high-temperature austenite in order to attain grain refinement via recrystallization. Refined austenite grain leads to refinement of ferrite grain, which improves mechanical properties after phase transformation via either isothermal or continuous cooling treatments. It is also well-known that high strength $\mathrm{V}$-steel is mainly attained by $\alpha / \gamma$-interphase VC precipitation in ferrite and pearlite. However, it should be noticed that the influences of vanadium on phase transformation, such as increasing ferrite fraction, elongated incubation time for pearlite transformation, and promotion of bainite transformation may ultimately change mechanical properties. For example, bainite transformation decreases strength due to diminishing of VC precipitation in V-steel.

An effective control on mechanical properties is significant and can be achieved by advanced understanding of microstructural evolution in during thermo-mechanical pro- cess. Since the mechanical properties of V-steels depend on their microstructures, such as alloying elements, ferrite and pearlite fractions, ferrite grain size, lamellar spacing of pearlite, and VC precipitate size and volume fraction, an accurate model to predict microstructural evolution is essential.

\section{Mathematical Models}

\subsection{Recrystallization}

Hot-deformed austenite basically proceeds to dynamic and static recrystallization. Estrin ${ }^{1)}$ reported that during a thermo-mechanical processing the geometrical dislocations accommodating the plastic deformation between grains. In addition, deformation-induced dislocation density is governed by two competing processes: work hardening (hard) and dynamic recovery (softening). The variation of dislocation density with respect to strain is proposed as

$$
\frac{d \rho}{d \varepsilon}=\left(k_{1} \cdot \sqrt{\rho}+\frac{1}{b \cdot d}\right)_{\text {hard }}-\left(k_{2} \cdot \rho\right)_{\text {soft }}
$$

The $\rho$ is dislocation density, $b$ the Burgers vector, $d$ the grain diameter, and $k_{1}$ and $k_{2}$ are parameters. The suffixes, hard and soft, represent work hardening and softening items in equation, respectively. In order to derive an equation to describe 
variation of dislocation density with respect to time, the work hardening item from Eq. (1) is transformed to

$$
\left(\frac{d \rho}{d t}\right)_{\text {hard }}=k_{1} \cdot \sqrt{\rho} \cdot\left(1+\frac{1}{b \cdot 2 \cdot r_{d r x} \cdot k_{1} \cdot \sqrt{\rho}}\right)_{\text {hard }} \cdot \frac{d \varepsilon}{d t}
$$

It was taken into consideration when fine-grained polycrystalline materials are involved, the mean free path of dislocation can be identified with the spacing between grain boundaries. For sake of simplicity, the increasing rate of dislocation density due to work hardening $(d \rho / d t)_{\text {hard }}$ is considered as a function of dynamic recrystallized grain radius $\left(r_{d r x}\right)$ and deformation rate $(d \varepsilon / d t)$. The dislocation density $(\rho)$ before deformation is regarded as constant. Hence, Eq. (2) is rewritten as

$$
\left(\frac{d \rho}{d t}\right)_{\text {hard }}=C_{1} \cdot\left(1+\frac{1}{\sqrt{2 \cdot r_{d r x}}}\right) \cdot \dot{\varepsilon}
$$

The parameter, $C_{l}$, is approximately fitted by $8.5 \times 10^{14}$, and $\dot{\varepsilon}$ is the deformation rate.

Elimination of dislocation density with time due to softening, $(d \rho / d t)_{\text {soft }}$, is transformed to

$$
\left(\frac{d \rho}{d t}\right)_{\text {soft }}=k_{2} \cdot \rho \cdot \frac{d \varepsilon}{d t}
$$

As above-mentioned, if $\rho$ is regarded as constant, the $(d \rho / d t)_{\text {soft }}$ is linearly related to deformation rate. However, the temperature effect on variation of dislocation density cannot be ignored. Dislocations are basically eliminated dramatically at high temperature. Based on Avrami model Lin et al. ${ }^{2)}$ reported equations to calculate the metadynamic recrystallized fraction of 42CrMo steel as shown follows

$$
\begin{gathered}
X_{\text {mdrec }}=1-\exp \left[-0.693\left(\frac{t}{t_{0.5}}\right)^{0.65}\right] \ldots \ldots . \\
t_{0.5}=7.1287 \times 10^{-9} \cdot \dot{\varepsilon}^{-0.5751} \exp \left(\frac{182288}{R \cdot T}\right)
\end{gathered}
$$

The $t_{0.5}$ represents time for $50 \%$ metadynamic recrystallization fraction. Although metadynamic recrystallization is not considered in the present model, according to Eq. (6) a relation between time for dynamic recrystallization and strain rate can be approximately defined as

$$
t=p \cdot\left(\frac{d \varepsilon}{d t}\right)^{-q} \cdot \exp \left(\frac{Q}{R \cdot T}\right)
$$

The $Q$ represents active energy, $R$ the gas constant, $T$ the absolute temperature, and $p, q$ are empirical fitting parameters. Eq. (7) is further approximated as

$$
\left(\frac{d \varepsilon}{d t}\right)=t^{-1 / q} \cdot p^{1 / q} \cdot \exp \left(\frac{-Q}{R \cdot T}\right)
$$

To put Eq. (8) into Eq. (4), the equation is then

$$
\left(\frac{d \rho}{d t}\right)_{\text {soft }}=k_{2} \cdot \rho \cdot t^{-1 / q} \cdot p^{1 / q} \cdot \exp \left(\frac{-Q}{R \cdot T}\right)
$$

Since $k_{2}, \rho, p, q$ are regarded as constants, Eq. (9) is rewritten as follows to describe the eliminated dislocation density due to dynamic recrystallization.

$$
\left(\frac{d \rho}{d t}\right)_{s o f t}=C_{2} \cdot t^{-1 / q} \cdot \exp \left(\frac{-Q}{R \cdot T}\right)
$$

The parameter $C_{2}$ and $q$ are approximated as 63 and 3.33, respectively. The active energy $Q$ is given as $66.5 \mathrm{~kJ} / \mathrm{mol}$. The variation of dislocation density with time described by Eq. (1) is rearranged as

$$
\frac{d \rho}{d t}=C_{1} \cdot \dot{\varepsilon} \cdot\left(1+\frac{1}{\sqrt{2 \cdot r_{d r x}}}\right)-C_{2} \cdot t^{-0.3} \cdot \exp \left(\frac{-Q}{R \cdot T}\right) \ldots \ldots .
$$

From Eq. (11), it can be seen that strain rate and dynamic grain size controls the variation of dislocation density by work hardening. The elimination of dislocation density is mainly controlled by temperature and time. Default value of minimum dislocation density is $10^{10} / \mathrm{m}^{2}$.

Dynamic recrystallized grain size $\left(r_{d r x}\right)$ decreases with increasing strain rate and decreasing deformation temperature. It can be expressed using power law function of ZenerHollomon parameter, Z, shown as following ${ }^{3-5)}$

$$
\begin{gathered}
r_{d r x}=C_{3} \cdot Z^{-q} \ldots . \\
Z=\dot{\varepsilon} \cdot \exp \left(\frac{Q}{R \cdot T}\right)
\end{gathered}
$$

Through combining Eqs. (12) and (13), the equation is transformed to

$$
r_{d r x}=C_{3} \cdot \dot{\varepsilon}^{-q} \cdot \exp \left(\frac{-q \cdot Q}{R \cdot T}\right)
$$

The $C_{3}$ and $q$ are materials dependent constants and approximated as 0.102 and 0.3043 , respectively. $Q$ is given as 25 $\mathrm{kJ} / \mathrm{mol}$.

When dislocation density reaches the critical dislocation density $\left(\rho_{c}\right)$ the dynamic recrystallization starts. The $\rho_{c}$ is approximated as a function of temperature and dynamic grain radius $\left(r_{d r x}\right)$ in the present work as

$$
\rho_{c}=C_{4} \cdot\left[1+\frac{1}{\sqrt{2 \cdot r_{d r x}}}\right] \cdot \exp \left(\frac{Q}{R \cdot T}\right)
$$

$C_{4}$ is the empirical parameter fitted by $2.125 \times 10^{10}$, and $Q$ is given as $90 \mathrm{~kJ} / \mathrm{mol}$.

Peczak and Luton ${ }^{6-8)}$ have considered that nucleation rate is a function of deformation temperature. They also investigated a linear relationship between nucleation rate and strain rate. Therefore, Ding and Guo ${ }^{9)}$ reported that nucleation rate for dynamic recrystallization, $d N / d t$, is a function of temperature and strain rate. The proposed equation is

$$
\frac{d N}{d t}=C_{4} \cdot \dot{\varepsilon} \cdot \exp \left(\frac{-Q}{R \cdot T}\right)
$$

The activation energy $(Q)$ is given as $250 \mathrm{~kJ} / \mathrm{mol}$ and the constant $C_{4}$ is approximated as $2 \times 10^{25}$ by fitting experimental data. In the present model, it is assumed that nucleation density increased only by deformation. The default value of minimum nucleation density is $10^{10} / \mathrm{m}^{3}$, i.e. this value also represents nucleation density for static recrystallization. Then, the mean grain radius of dynamic and static recrystallized grain radius at time $t_{i}, r_{\text {ave }}\left(t_{i}\right)$, is obtained by

$$
r_{\text {ave }}\left(t_{i}\right)=\left\{\frac{3}{4 \cdot \pi \cdot\left[N\left(t_{i-1}\right)+d N\left(t_{i}\right)\right]}\right\}^{1 / 3}
$$

In the case of steel without vanadium addition (V-free steel), the mean recrystallized grain size can be calculated by Eq. (17). However, in the microalloyed steels, such as V- 
steels, the precipitates interact with grain boundaries and retard boundary mobility. In consequence, recrystallization is stopped, so that the recrystallized grain radius will be treated as: ${ }^{10,11)}$

$$
r_{a v e}^{\prime}=C_{5} \cdot \frac{r_{v c}}{f_{v c}}
$$

where $r_{v c}$ and $f_{v c}$ is respectively the radius and volume fraction of VC. The constant $C_{5}$ is approximately estimated as 0.44 . The values of $f_{v c}$ and $r_{v c}$ are determined using the model for predicting VC precipitation developed by Miyamoto and Furuhara. ${ }^{12)}$ In this model, the variation of $r_{v c}$ and $f_{v c}$ depends on dislocation density and temperature, as well as vanadium and carbon concentrations. Therefore, when $r_{\text {ave }}^{\prime}>r_{\text {ave }}$, the $r_{\text {ave }}$ will be the recrystallized grain size rather than $r_{\text {ave }}$.

Vanadium addition effects on recrystallization behavior have been considered: 1 . solute-drag effect on grain boundary mobility; 2 . VC-pinning effect $\left(F_{\text {pin }}\right)$ on grain growth. Solute-drag effect of microalloying elements on grain boundary mobility has been proposed by Cahn. ${ }^{13)}$ The solute-drag effect reduces the mobility in a non-linear manner. Zurob et al. ${ }^{14)}$ have simplified Cahn's equation via dealing with boundary mobility as a constant and proportional to solute content. For a low driving force, Cahn's solution has been simplified to be

$$
\frac{1}{M(t)}=\frac{1}{M_{\text {pure }}}+\alpha \cdot X_{\text {solute }}
$$

Even in a pure material, the estimation of a standard mobility is a difficult problem. We attempt to simplify the mobility as a function of both temperature and solute content. The boundary mobility is decreased when the temperature is decreased or the vanadium addition is increased due to the solute-drag effect. The equations can be described as

$$
\begin{gathered}
M_{S 45 C}=C_{6} \cdot \exp \left(\frac{-Q}{R \cdot T}\right) \ldots \ldots \ldots \ldots \ldots \ldots . . . \\
M_{S 45 C+X v}=\left(\frac{1}{M_{S 45 C}}+C_{7} \cdot X v \cdot M_{S 45 C}\right)^{-1} .
\end{gathered}
$$

where $M_{S 45 C}$ is the intrinsic grain boundary mobility of medium carbon steel $(S 45 C)$, and $X_{V}$ the molar fraction of vanadium. The value of $Q$ is given the same value of $282 \mathrm{k}$ $\mathrm{J} / \mathrm{mol}$. The parameters $C_{6}$ and $C_{7}$ are approximated as 1.8 and 80 , respectively.

The kinetics for recrystallization is identical to a driving force, $F_{\text {strain }}$, induced by stored dislocation after deformation. However, during recrystallization the $F_{\text {strain }}$ will be diminished by precipitate pinning force, $F_{\text {pin }}$. When $F_{\text {strain }}$ is lower than $F_{p i n}$, the recrystallization is stopped. Since we neglect static recovery the driving force for recrystallization, $F_{\text {rex }}$, can be simply expressed as

$$
\begin{array}{r}
F_{\text {rex }}=F_{\text {strain }}-F_{\text {pin }} \\
F_{\text {strain }}=\left[\rho \cdot \mu \cdot b^{2}\right] / 2 \ldots \ldots \ldots \ldots \ldots \ldots \ldots \ldots \ldots \ldots \ldots \ldots \ldots \ldots \ldots \ldots \\
\mu[\mathrm{Pa}]=\left(8.1 \times 10^{10}\right) \cdot\left(0.91-\frac{T-300}{1810}\right) .
\end{array}
$$

The $\rho$ is the strain-induced dislocation density obtained by Eq. (11); $\mu[\mathrm{Pa}]$ the shear modulus as a function of absolute temperature, and Burgers vector $(b)$ is given as $0.25 \times 10^{-9} \mathrm{~m}$. Smith ${ }^{15)}$ has investigated the pinning of grain boundaries by particles in many ways and proposed the formula as

$$
F_{p i n}=\frac{3 \cdot E_{v c} \cdot f_{v c}}{2 \cdot r_{v c}}
$$

The $E_{v c}$ is VC surface energy and given as $0.4 \mathrm{~J} / \mathrm{m}^{2}$. Assuming the recrystallization is in spherical shape, the total volume of recrystallization at time $t_{i}$ is considered as

$$
V\left(t_{i}\right)=\frac{4 \pi}{3} \cdot\left[r_{\text {rex }}\left(t_{i-1}\right)+M\left(t_{i}\right) \cdot F_{r e x}\left(t_{i}\right) \cdot\left(t_{i}-t_{i-1}\right)\right]^{3} \ldots
$$

The $M\left(t_{i}\right)$ and $F_{\text {rex }}\left(t_{i}\right)$ represents respectively the grain boundary mobility and driving force for recrystallization at time $t_{i}$. The volume fraction of recrystallization at time $t_{i}$ is then

$$
X_{\text {rex }}\left(t_{i}\right)=\sum_{i=1}^{n} V\left(t_{i}\right) \cdot N\left(t_{i}\right)
$$

Various studies ${ }^{16,17)}$ have been mentioned that the optimized grain growth is given by

$$
\begin{array}{r}
r_{i+1}=\sqrt[n]{\left(r_{i}\right)^{n}+M \cdot E_{G . B} \cdot P \cdot d t} \ldots \\
P=1-\left(\frac{F_{p i n} \cdot r_{\text {ave }}}{2 \cdot E_{G . B}}\right) \ldots \ldots \ldots . . . \\
E_{G . B}\left[J / m^{2}\right]=1.3-\left(5 \times 10^{-4}\right) \cdot T
\end{array}
$$

$P$ is the effective factor of VC. Without VC in austenite, $P$ equals to 1 for grain growth of $\mathrm{V}$-free steel. $M$ is the grain boundary mobility, $E_{G . B}$ the grain boundary energy, $r_{\text {ave }}$ the mean radius of static and dynamic recrystallized grain. The $n$ is materials dependent parameter. When $n$ is given as 2 , the calculated austenite grain radius changing with time for $\mathrm{S} 45 \mathrm{C}$ are in a good agreement with experimental data, as shown in Fig. 1.

\subsection{Phase Transformation}

\subsubsection{Thermodynamic Data}

Thermodynamic data of $\mathrm{Fe}-\mathrm{C}-\mathrm{Si}-\mathrm{Mn}-\mathrm{V}$ system associated with equilibrium compositions and driving force are essential requirements for the theoretical simulation of transformation kinetics. In the present study the equation for these thermodynamic data under para-equilibrium were calculated by commercial "Thermocalc" software.

The para-equilibrium carbon concentration with the

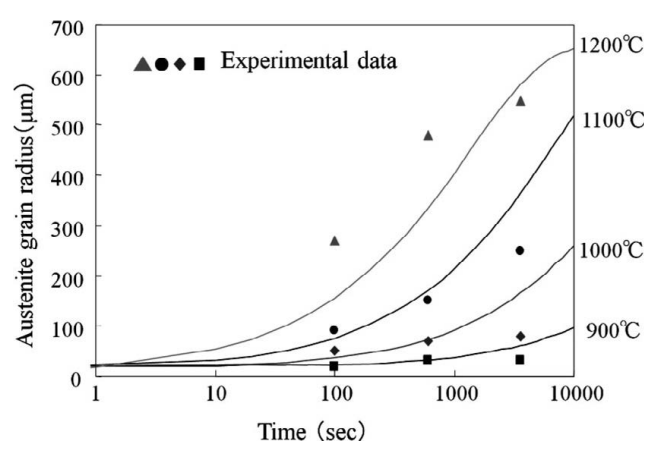

Fig. 1. Calculated and experimental results of austenite grain radius changing with time in $\mathrm{S} 45 \mathrm{C}$ at different temperatures. 


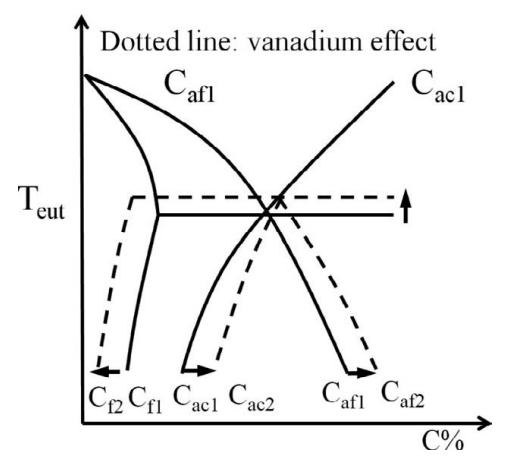

Fig. 2. Pseudo-diagram of $\mathrm{Fe}-\mathrm{C}$ system with effect of vanadium addition.

influence of vanadium addition is illustrated in Fig. 2. The $C_{f 2}, C_{a f 2}$ and $C_{a c 2}$ represents respectively the pseudo-ferrite, -austenite/ferrite and -austenite/cementite interface due to vanadium addition. The vanadium addition effect on eutectoid temperature $\left(T_{\text {eut }}\right)$ is calculated as

$$
\begin{array}{r}
T_{\text {eut }}=1026-749 \cdot C_{a}+1.667 \cdot X_{V} \\
C_{a}=\frac{X_{C}-X f \cdot C_{f}}{1-X f} \ldots \ldots \ldots \ldots
\end{array}
$$

$C_{a}$ and $C_{f}$ are the para-equilibrium carbon contents in austenite and ferrite, respectively. $X_{C}$ is the carbon content of steel and $X_{V}$ for vanadium. The $X f$ is then the ferrite volume fraction.

The undercooling $(\Delta T)$ for calculating pearlite fraction $\left(X_{p}\right)$ and lamellar spacing $(S)$ in the prediction of pearlite transformation is

$$
\Delta T=T_{e u t}-T
$$

The driving force for ferrite nucleation from austenite, in which the carbon and vanadium contents are taken into account, is obtained using 'ThermoCalc' software as well.

\subsubsection{Ferrite Transformation}

Ferrite which grows by a diffusional mechanism can be classified into two main forms: allotriomorphic ferrite and idiomorphic (or intragranular) ferrite. ${ }^{18)}$

\subsubsection{Ferrite Nucleation}

The nucleation rate $(J)$ is given by the classical theory of nucleation as

$$
J=N_{v} \cdot \beta^{*} \cdot Z \cdot \exp \left(-\frac{d G_{v}}{B \cdot T}\right) \cdot \exp \left(-\frac{\tau}{t}\right)
$$

where $N_{v}$ is the density of nucleation sites, $\beta^{*}$ the rate at which the atoms of iron are being added to the critical nucleus, $Z$ the Zeldovich non-equilibrium factor, $d G_{v}$ the driving force for ferrite nucleation from austenite, $B\left(=1.38 \times 10^{-23} \mathrm{~J} / \mathrm{K}\right)$ the Boltzmann constant, $T$ the absolute temperature in $K, \tau$ the incubation time of nucleation, and $t$ is the time in second.

Lange et al. ${ }^{19)}$ proposed a model to calculate the classical nucleation rate of allotriomorphic ferrite based on a traditional disk-shaped pillbox nucleus. Based on this model, the incubation time for ferrite nucleation was approximated as:

$$
\tau=\frac{\left(8 \times 10^{5}\right) \cdot B \cdot T \cdot a^{4} \cdot E}{D_{c} \cdot C_{a} \cdot V_{a}^{2} \cdot \Delta G_{v}^{2}}
$$

And the value of $\beta^{*} \cdot Z$ will be approximated as

$$
\beta^{*} \cdot Z=\frac{2 \cdot D_{C} \cdot C_{a} \cdot V_{a}}{a^{4}} \cdot \sqrt{\frac{E}{B \cdot T}}
$$

where $D_{c}$ is the diffusivity of carbon in austenite, $V_{a}$ $\left(=1.16 \times 10^{-29} \mathrm{~m}^{3}\right)$ the volume of an iron atom in austenite, $a$ $\left(=1.82 \times 10^{-10} \mathrm{~m}\right)$ the lattice parameters of austenite; $C_{a}$ the average carbon content in mole fraction; $\Delta G_{v}$ the change of volume free energy associated with the formation of nucleus. The value of surface energy $(E)$ is $0.021 \mathrm{~J} / \mathrm{m}^{2}$ for nuclei at grain boundary $\left(E_{\text {gra }}\right), 0.018 \mathrm{~J} / \mathrm{m}^{2}$ at grain corner $\left(E_{\text {corner }}\right)$, and $0.015 \mathrm{~J} / \mathrm{m}^{2}$ at inner grain $\left(E_{\text {inc }}\right)$.

The $N_{v}$ at grain boundary $\left(N_{v \_ \text {gra }}\right)$, grain corner $\left(N_{v \_ \text {corner }}\right)$ and grain interior $\left(N_{v_{-} \text {intra }}\right)$ were derived as the functions of austenite grain size and approximated as following

$$
\begin{array}{r}
N_{v_{-} \text {gra }}=\frac{3}{d_{a}} \ldots \ldots \ldots \ldots \ldots \ldots \ldots \ldots \ldots \ldots \ldots \\
N_{v_{-} \text {corner }}=\frac{11.5}{d_{a}^{3}} \ldots \ldots \ldots \ldots \ldots \\
N_{v_{-} \text {inc }}=10^{8}+\frac{2.5 \times 10^{7}}{d_{a}{ }^{2}} \cdot \frac{X_{V}}{0.0054}
\end{array}
$$

where $d_{a}$ is the austenite grain size in meter, and $X_{V}$ is the vanadium content in molar fraction.

Putting Eqs. from (35) to (39) into Eq. (34), the nucleation rate at grain corner $\left(J_{v_{-} \text {corner }}\right)$, grain boundary $\left(J_{v_{\text {gra }}}\right)$ and grain interior $\left(J_{v_{-} i n c}\right)$ were respectively approximated as

$$
J_{v_{-} \text {corner }}=\left(3 \times 10^{-8}\right) \cdot N_{v_{-} \text {corner }} \cdot \beta^{*} \cdot Z \cdot \exp \left(-\frac{16 \cdot \pi \cdot E_{\text {corner }}{ }^{3}}{3 \cdot d G_{v} \cdot B \cdot T}\right)
$$

$$
J_{v_{-} g r a}=\left(1 \times 10^{-3}\right) \cdot N_{v_{-} g r a} \cdot \beta^{*} \cdot Z \cdot \exp \left(-\frac{16 \cdot \pi \cdot E_{g r a}{ }^{3}}{3 \cdot d G_{v} \cdot B \cdot T}\right)
$$

$$
J_{v_{-} i n c}=\left(3 \times 10^{-24}\right) \cdot N_{v_{-} i n c} \cdot \beta^{*} \cdot Z \cdot \exp \left(-\frac{16 \cdot \pi \cdot E_{i n c}{ }^{3}}{3 \cdot d G_{v} \cdot B \cdot T}\right)
$$

\subsubsection{Parabolic Rate Constant}

The growth rate of ferrite was derived by Zener and the radius of ferrite at time $t$ is given by

$$
R(t)=\alpha \cdot \sqrt{t-\tau}
$$

where $\alpha$ is the parabolic rate constant; $t$ the holding time, and $\tau$ is the incubation time.

Bradley et $a{ }^{20)}$ proposed that allotriomorphic ferrite grains grow as ellipsoid with the aspect ratio of $3: 1$, then

$$
R(t)=\frac{\alpha \cdot \sqrt{(t-\tau)}}{3}
$$

The parabolic rate constant, $\alpha$, is basically described as

$$
\alpha=\frac{C_{a}-C_{0}}{\sqrt{\left(C_{a}-C_{f}\right) \cdot\left(C_{0}-C_{f}\right)}} \cdot \sqrt{D_{c} \cdot t}
$$

where $D_{c}$ is the diffusivity of carbon and $t$ is the time in second. The $C_{a}$ is the carbon concentration in austenite at aus- 
tenite/ferrite interface; $C_{f}$ the carbon concentration in ferrite at interface; $C_{0}$ the carbon concentration in austenite far from ferrite. The equilibrium carbon in diffusion field at austenite side is calculated by $\left(C_{a}+C_{0}\right) / 2$.

During ferrite transformation in microalloyed steel, two effective factors to the transformation are usually considered, i.e. solute-drag and precipitate-pinning effects. Back to Fig. 2, since the influence of vanadium addition on paraequilibrium of carbon, including carbon in ferrite $\left(C_{f 2}\right)$, austenite-ferrite interface $\left(C_{a f 2}\right)$ and austenite-cementite interface $\left(C_{a c}\right)$, has been considered and calculated using ThermoCalc, the solute-drag effect of vanadium on ferrite transformation can be neglected. On the other hand, we are interested in the influence of interphase VC precipitation on ferrite transformation. Considering the partition of carbon occurs before interphase VC precipitation, coupled with a gradual build-up. The vanadium is fully consumed by VC and the carbon at austenite-ferrite interface depleted by $\mathrm{VC}$ is defined as $C_{v c}$. The carbon diffusivity at interface is therefore accelerated. Eventually, the carbon depletion advances the ferrite formation sufficiently to drag the austenite-ferrite interface away, and the partitioning process recommences. With effect of vanadium addition, the Eq. (45) is then rewritten as

$$
\alpha^{\prime}=\frac{\left(C_{a}-C_{0}\right) \cdot \sqrt{D_{C} \cdot t}}{\sqrt{\left(C_{a}-C_{f}-C_{v c}\right) \cdot\left(C_{0}-C_{f}-C_{v c}\right)}}
$$

Kaufman et $a l^{21)}$ proposed a model to calculate the diffusivity of carbon in austenite, $D_{c}\left[\mathrm{~m}^{2} / \mathrm{s}\right]$, as a function of carbon in austenite. The modified model used in the present study is

$$
\begin{aligned}
& D_{c}\left[m^{2} / s\right]=\left(5 \times 10^{-5}\right) \cdot \exp \left(-33.2 \cdot C_{a}\right) . \\
& \exp \left[\frac{-10^{5} \cdot\left(1.52-4.2 \cdot C_{a}-26 \cdot C_{a}{ }^{2}\right)}{R \cdot T}\right] . .
\end{aligned}
$$

It is known that the growth rate of pro-eutectoid ferrite is inhibited by the drag-effect of alloying elements and pinning effect of VC particle. According to our results, ferrite transformation rate is inhibited mainly by the pinning effect of $\mathrm{VC}$ at temperatures lower than $675^{\circ} \mathrm{C}$ since the vanadium is consumed very rapid for the interphase VC precipitation. The pinning force of $\mathrm{VC}$ to the driving force of ferrite transformation can be obtained by

$$
\Delta G_{p i n}=\frac{2 \cdot E_{v c} \cdot V_{m} \cdot f_{v c}}{2 \cdot r_{v c}}
$$

where $E_{v c}$ is the interfacial energy of $\mathrm{VC}$ and $V_{m}$ is the molar volume of iron.

\subsubsection{Mean Diameter of Ferrite Grain}

The volume fraction of pro-eutectoid ferrite in austenite is equal to the summation of allotriomorphic and intragranular fractions. It can be simply described as

$$
\begin{gathered}
X f=X f_{\text {allo }}+X f_{\text {intra }} \\
X f=\frac{4 \pi}{3} \cdot\left(\frac{d f}{2}\right)^{3} \cdot\left(N_{\text {allo }}+N_{\text {intra }}\right) \\
X f_{\text {allo }}=\frac{4 \pi}{3} \cdot\left(\frac{d f_{\text {allo }}}{2}\right)^{3} \cdot N_{\text {allo }}
\end{gathered}
$$

$$
X f_{\text {intra }}=\frac{4 \pi}{3} \cdot\left(\frac{d f_{\text {intra }}}{2}\right)^{3} \cdot N_{\text {intra }}
$$

Putting Eqs. from (50) to (52) into Eq. (49), the mean diameter of ferrite, $d f$, can be obtained by

$$
d f=\left[\frac{\left(d f_{\text {allo }}\right)^{3} \cdot N_{\text {allo }}+\left(d f_{\text {intra }}\right)^{3} \cdot N_{\text {intra }}}{N_{\text {allo }}+N_{\text {intra }}}\right]^{1 / 3}
$$

The $N_{\text {allo }}$ and $N_{\text {intra }}$ represent the density of allotriomorphic and intragranular ferrite grain, respectively. The $d f_{\text {allo }}$ is the mean diameter of allotriomorphic ferrite grain, and the $d f_{\text {intra }}$ for intragranular one.

The allotriomorphic ferrite fraction is schematically shown in Fig. 3. During ferrite transformation at time $t_{i}$, allotriomorphic ferrite grains in diameter of $d f_{\text {allo }}$ and initial grains growing from nuclei coexist at grain boundaries. The radius of initial grain is calculated by $\alpha \cdot\left(t_{i}-t_{i-1}\right)^{1 / 2}$ and the number of initial grain is regarded as the increment of allotriomorphic grains in the time interval of $t_{i}-t_{i-l}$. Then, $N_{\text {allo }}\left(t_{i}\right)$ is defined as the number of allotriomorphic ferrite grains existing at grain boundaries at time $t_{i}$, and $d N_{\text {allo }}\left(t_{i}\right)$ is the increment of grain number during $t_{i}-t_{i-l}$. The mean diameter of allotriomorphic ferrite, $d f_{\text {allo }}$, is described as

$$
d f_{\text {allo }}=\frac{d f_{\text {allo }}\left(t_{i}\right) \cdot N_{\text {allo }}\left(t_{i}\right)+\left(2 \cdot \alpha \cdot \sqrt{t_{i}-t_{i-1}}\right) \cdot d N_{\text {allo }}\left(t_{i}\right)}{N_{\text {allo }}\left(t_{i}\right)+d N_{\text {allo }}\left(t_{i}\right)}
$$

The illustration of allotriomorphic grain size at time $t_{i}$, $d f_{l l o}\left(t_{i}\right)$, is shown in Fig. 4. Then

$$
d f_{\text {allo }}\left(t_{i}\right)=d f_{\text {allo }}\left(t_{i-1}\right)+2 \cdot \alpha \cdot \sqrt{t_{i}-t_{i-1}}
$$

where $\alpha$ is the parabolic rate constant.

Ferrite fraction is basically described using Johnson-

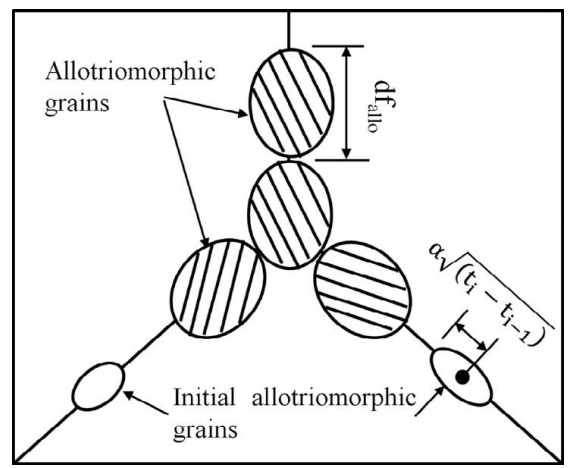

Fig. 3. Illustration of allotriomorph ferrite in a unit volume of austenite.

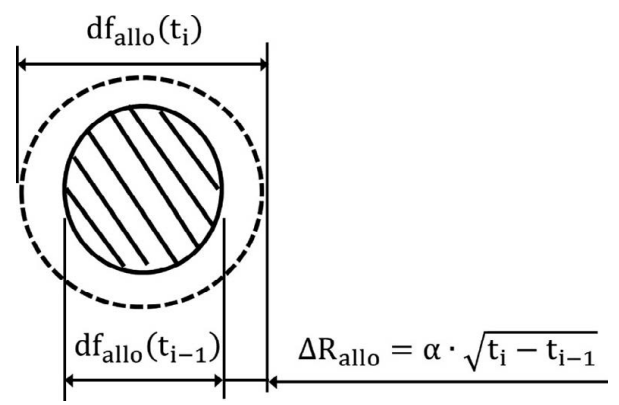

Fig. 4. Illustration of allotriomorph size at time $t_{i}$. 
Mehl-Avrami (J-M-A) ${ }^{22)}$ equation as follows

$$
X=1-\exp \left[-k \cdot(t-\tau)^{n}\right]
$$

where $X$ is the transformed volume fraction; $k$ the coefficient depends on nucleation and growth rate; $t$ the holding time; $\tau$ the incubation time, and $n$ is the materials depending constant.

The volume fraction of allotriomorphic ferrite, $X f_{\text {allo }}\left(t_{i}\right)$, in our present work is described as follows:

$$
X f_{\text {allo }}\left(t_{i}\right)=\left\{1-\exp \left[-V_{\text {allo }}\left(t_{i-1}\right)-V_{\text {allo }}\left(t_{i}\right)\right]\right\}
$$

where $N_{v \text { gra }}$ is the ferrite nuclei density at grain boundary. The shape of allotriomorph grain is assumed spherical, then the volumes of allotriomorph ferrite at time $t_{i}, V_{\text {allo }}\left(t_{i}\right)$, and at time $t_{i-l}, V_{\text {allo }}\left(t_{i-1}\right)$, in the unit volume of austenite are

$$
\begin{array}{r}
V_{\text {allo }}\left(t_{i-1}\right)=N_{\text {allo }}\left(t_{i-1}\right) \cdot \frac{4 \pi}{3} \cdot\left[\frac{d_{\text {allo }}\left(t_{i-1}\right)}{2}\right]^{3} \ldots \ldots . \\
V_{\text {allo }}\left(t_{i}\right)=d N_{\text {allo }}\left(t_{i}\right) \cdot \frac{4 \pi}{3} \cdot\left[\frac{d f_{\text {allo }}\left(t_{i-1}\right)}{2}+\alpha \cdot \sqrt{t_{i}-t_{i-1}}\right]^{3} .
\end{array}
$$

As well as the approach to allotriomorph, the volume fraction of intragranular ferrite at time $t_{i}$ is described as

$$
X f_{\text {intra }}\left(t_{i}\right)=V_{\text {intra }}\left(t_{i-1}\right) \cdot N_{\text {intra }}\left(t_{i-1}\right)+V_{\text {intra }}\left(t_{i}\right) \cdot d N_{\text {intra }}\left(t_{i}\right)
$$

The $N_{\text {intra }}\left(t_{i-1}\right)$ represents the number of intragranular ferrite at time $t_{i-1}$, and $d N_{\text {intra }}\left(t_{i}\right)$ the increment of grain number at time $t_{i}$. The $V_{\text {intra }}\left(t_{i-1}\right)$ is the mean volume of intragranular ferrite at time $t_{i-1}$, and $V_{\text {intra }}\left(t_{i}\right)$ for the one at time $t_{i}$. As assumed before, the intragranular ferrite radius is $1 / 3$ of longer radius of allotriomorph, the $V_{\text {intra }}\left(t_{i-1}\right)$ and $V_{\text {intra }}\left(t_{i}\right)$ is respectively defined as

$$
\begin{gathered}
V_{\text {intra }}\left(t_{i-1}\right)=\frac{4 \pi}{3} \cdot\left[\frac{d f_{\text {intra }}\left(t_{i-1}\right)}{2}\right]^{3} \ldots . . \\
V_{\text {intra }}\left(t_{i}\right)=\frac{4 \pi}{3} \cdot\left[\frac{d f_{\text {intra }}\left(t_{i-1}\right)}{2}+\frac{\alpha \cdot \sqrt{\left(t_{i}-t_{i-1}\right)}}{3}\right]^{3}
\end{gathered}
$$

The mean volume of intragranular ferrite, $V_{\text {intra }}$, can be described in the other way as

$$
V_{\text {intra }}=\frac{4 \pi}{3} \cdot\left[\frac{d f_{\text {intra }}}{2}\right]^{3}
$$

Then, the mean diameter of intragranular ferrite, $d f_{\text {intra }}$, is simply derived from Eq. (63) as

$$
d f_{\text {intra }}=\left[\frac{6 \cdot V_{\text {intra }}}{\pi}\right]^{1 / 3}
$$

\subsubsection{Pearlite Transformation}

The pearlite growth involves the carbon diffusion in austenite from a high concentration at the ferrite-austenite interface to a low concentration at the cementite-austenite interface. Both ferrite and cementite must be present at the boundary, and it is clear that the growth of each must be continuous if the velocity is to remain constant.

Mehl et al. ${ }^{23-27)}$ established the mechanism of pearlite growth. The mechanism reveals that rate of transformation from austenite to pearlite is determined by pearlite nucleation and growth rate. However, in present model predicting phase transformation of $\mathrm{V}$-steel the pearlite growth is assumed to start right after ferrite transformation. Based on this assumption, site saturation can be ignored and pearlite growth rate is taken into account for pearlite transformation. Instead, Brandt model is used. Brandt ${ }^{28)}$ assumed that Mehl's mechanism is substantially correct and proposed a model to calculate the edgewise velocity of pearlite growth $(V)$ and growth rate $\left(G_{p}\right)$. It is also assumed that lamellar structure must be established before $V$ and $G_{p}$ can achieve a constant. The model is described as follows

$$
\begin{gathered}
V=\frac{4 \cdot \pi \cdot D}{S \cdot \sqrt{P^{2}-1}} \ldots . \\
P-N \cdot \frac{\frac{2}{L^{1+P}}+L}{M+\frac{2}{L^{1+P}}}=1 \\
N=2 \cdot \frac{C_{c}-C_{a}}{C_{a}-C_{a c}} \ldots \\
L=\frac{C_{a}-C_{f}}{C_{c}-C_{a}} \ldots \ldots . \\
M=\frac{C_{a f}-C_{a}}{C_{a}-C_{a c}} \ldots . . .
\end{gathered}
$$

$C_{a f}$ is defined as the carbon concentration in austenite at the center of the ferrite-austenite interface. $C_{a c}$ is the carbon concentration in austenite at the center of the cementite-austenite interface. $C_{c}$ is the carbon concentration in cementite and $C_{f}$ as the carbon concentration in ferrite.

The overall pearlite transformation rate can be described by K-J-M-A theory, which predicts the pearlite fraction as a function of isothermal transformation time $t$. The increment of pearlite fraction is then described as

$$
\begin{array}{r}
d X_{P}=\left[1-\exp \left(-G_{P} \cdot(t+d t)\right)\right]-\left[1-\exp \left(-G_{P} \cdot t\right)\right] \ldots . . \\
G_{P}=V \cdot\left[1-\exp \left(-300 \cdot\left(C_{a}-C_{a c}\right)\right)\right] \cdot k \ldots \ldots . .
\end{array}
$$

where $k$ is the materials parameter as a function of undercooling and vanadium addition. It is empirically expressed as

$$
k=1-\frac{0.9}{1+\exp \left[-\left(\Delta T-2.9 \times 10^{6} \cdot X_{V}^{2}+3.4 \times 10^{4}+250\right]\right.}
$$

The lamellar spacing $(S)$ is basically reciprocal to undercooling $(\Delta T)$ and carbon concentration in austenite $\left(C_{a}\right)$. It is empirically derived in the present work as

$$
S=1.45 \times 10^{-5} \cdot\left(\frac{0.034}{C_{a}}\right) \cdot\left(\frac{1}{\Delta T}\right)
$$

The mean lamellar spacing at time $\mathrm{t}_{\mathrm{i}}, S\left(t_{i}\right)$, can be obtained by

$$
S\left(t_{i}\right) \cdot\left[X p\left(t_{i-1}\right)+d X p\left(t_{i}\right)\right]=S\left(t_{i-1}\right) \cdot X p\left(t_{i-1}\right)+S \cdot d X p\left(t_{i}\right)
$$

where $X_{p}\left(t_{i-1}\right)$ and $S\left(t_{i-1}\right)$ are the pearlite fraction and mean lamellar spacing at time $t_{i-l}$, respectively. 


\subsection{Yield Strength}

Gladman et al. ${ }^{29)}$ proposed an equation to describe the effects of ferrite and pearlite on the yield strength as

$$
\sigma_{Y P}=X f^{n} \cdot \sigma_{F}+\left(1-X f^{n}\right) \cdot \sigma_{P}
$$

where $\sigma_{Y P}$ is the yield strength of ferrite-pearlite aggregate, $\sigma_{F}$ the yield strength of ferrite, and $\sigma_{p}$ the yield strength of pearlite. The index ' $n$ ' is included in order to permit the yield strength to vary with pearlite content in a non-linear manner.

Based on an extension of the Hall-Petch ${ }^{30)}$ relationship, we developed an empirical function of yield strength for ferrite as

$$
\sigma_{F}=115.58+53.4 \cdot W_{s i}+4.28 \cdot W_{M n}+\frac{0.58}{\sqrt{d f}}
$$

where $W_{S i}$ and $W_{M n}$ are the silicon and manganese weight percent, respectively, and $d f$ is the ferrite grain size.

Gensamer $^{31)}$ suggested that the yield strength of pearlite depends on the logarithm of interlamellar spacing. However, Gladman et al. ${ }^{29)}$ suggested that relationship is supposed to be a linear dependence on $\mathrm{S}^{-1 / 2}$ based on their massive experimental evidence. In the present work, the yield strength of pearlite is empirically derived as

$$
\begin{gathered}
\sigma_{P}=109.9+1726 \cdot f_{\theta}+\frac{4.2 \times 10^{5}}{S} \ldots \ldots \\
f_{\theta}=\left(\frac{1.946 \cdot W_{C}}{12.72+0.046 \cdot W_{C}}\right) \cdot\left(\frac{X f+X p}{X p}\right) .
\end{gathered}
$$

where $f_{\theta}$ is the cementite fraction in pearlite, and $W_{C}$ is the weight percent of carbon.

For the precipitation strengthening, the interaction between hard particle (unshearable) and dislocation is generally explained by Orowan theory. ${ }^{32)}$ That is, when the moving dislocation meets a particle, the dislocation will be bowed-out caused by the pinning of hard particle. With increasing the applied stress, the moving dislocation will pass through the particle and leave behind a loop around it.

Ashby $^{33)}$ further developed the equation to take into account the interparticle spacing $(L)$, and the effect of statistically distributed particles, including particles volume fraction $\left(f_{v c}\right)$ and radius $\left(r_{v c}\right)$ on the applied stress $(\Delta \sigma)$. The Ahsby-Orowan relationship used in the present work is given as

$$
\begin{aligned}
& \Delta \sigma=0.84 \cdot M \cdot\left(\frac{1.2 \cdot G \cdot b}{2 \cdot \pi \cdot L}\right) \cdot \ln \left(k \cdot \frac{X}{2 \cdot b}\right) \ldots \ldots . . \\
& L=\sqrt{\frac{2 \cdot \pi \cdot r_{v c}^{2}}{3 \cdot f_{v c}}}
\end{aligned}
$$

where $\Delta \sigma$ is the increase in yield strength (MPa), $G$ is the shear modulus $\left(83 \times 10^{3} \mathrm{MPa}\right), b$ is the Burgers vector (= $\left.0.248 \times 10^{-9} \mathrm{~m}\right), f_{v c}$ is the volume fraction of $\mathrm{VC}, r_{v c}$ is the radius of $\mathrm{VC}$, and $X$ is the real (spatial) diameter of the particles in meter, $X=2 r_{v c}$. The $\mathrm{k}$ was estimated as 1 for VC precipitation strengthening in pearlite, and as 2.7 in ferrite.

Based on Gladman's equation accompanying with AshbyOrowan equation for $\mathrm{VC}$ precipitation strengthening, the equation is derived in the present work as

$$
\sigma_{Y P}=\left(\sigma_{F}+\Delta \sigma_{F}\right) \cdot\left(\frac{X f}{X f+X p}\right)^{n}+\left(\sigma_{P}+\Delta \sigma_{P}\right) \cdot\left(\frac{X p}{X f+X p}\right)^{n}
$$

where ' $n$ ' was empirically fitted as $1 / 3$. The $\Delta \sigma_{F}$ and $\Delta \sigma_{P}$ represent $\mathrm{VC}$ precipitation strengthening in ferrite and pearlite, respectively.

\section{Experimental Procedure}

Five carbon steels with different vanadium addition, $\mathrm{S} 10 \mathrm{C}+0.3 \mathrm{~V}, \mathrm{~S} 45 \mathrm{C}, \mathrm{S} 45 \mathrm{C}+0.3 \mathrm{~V}, \mathrm{~S} 75 \mathrm{C}$, and $\mathrm{S} 75 \mathrm{C}+0.3 \mathrm{~V}$ were prepared for the experiments. Their chemical compositions are shown in Table 1.

The steels were austenitized at $1200^{\circ} \mathrm{C}$ for $10 \mathrm{~min}$, and then deformed by compression at different temperature at range of $900-1200^{\circ} \mathrm{C}$ or without forging process. Following post-treatment is isothermal transformation at temperatures of $550-700^{\circ} \mathrm{C}$ for $600-14400 \mathrm{~s}$.

Specimens for microstructural quantification were mechanically polished and etched with $0.1 \%$ Nital solution, and then observed by optical microscopy (NIKON Eclipse Me600) and scanning electron microscopy (JOEL JSM6500F). Afterwards, ferrite fraction and pearlitic lamellar spacing were quantified. Tensile tests were carried out at room temperature using a Shimadzu AGS machine with a clip-on type extensometer for measuring strain.

\section{Comparison with the Present Model and Experimen- tal Data}

The modeling recrystallization of $\mathrm{S} 45 \mathrm{C}+0.3 \mathrm{~V}$ is shown in Fig. 5. The steels were austenitized at $1200^{\circ} \mathrm{C}$ for $10 \mathrm{~min}$, and then forged with $20 \%$ deformation in strain rate of $10 / \mathrm{s}$ (see Fig. 5(a)). The deformation temperatures are 1150, 1050 and $1000^{\circ} \mathrm{C}$. The deformed steels were hold at the temperature for different periods of time. The calculated and experimental results of recrystallized fraction and recrystallized grain size are shown in Figs. 5(b) and 5(c), respectively.

According to the study by Miyamoto and Furuhara, ${ }^{11)}$ for a $\mathrm{S} 45 \mathrm{C}+0.3 \mathrm{~V}$ steel the retained $\mathrm{VC}$ is almost solid-solutioned in austenite as austenitization temperature is higher than $1150^{\circ} \mathrm{C}$, i.e. $\mathrm{VC}$ precipitation in austenite occurs as temperature is lower than $1150^{\circ} \mathrm{C}$. As revealed in Fig. 5(b), the recrystallization kinetics at $1150^{\circ} \mathrm{C}$ is very rapid and $\mathrm{VC}$ precipitation in austenite does not occur to inhibit recrystallization and grain growth. As deformation temperature decreases to temperatures lower than $1150^{\circ} \mathrm{C}$, VC precipitation prefers to occur at deformation-induced dislocations. $\mathrm{VC}$ precipitates in austenite eventually retard the recrystallization and even stop it.

Table 1. Chemical composition of steels (wt $\%)$.

\begin{tabular}{lccccc}
\hline & $\mathrm{S} 10 \mathrm{C}+0.3 \mathrm{~V}$ & $\mathrm{~S} 45 \mathrm{C}$ & $\mathrm{S} 45 \mathrm{C}+0.3 \mathrm{~V}$ & $\mathrm{~S} 75 \mathrm{C}$ & $\mathrm{S} 75 \mathrm{C}+0.3 \mathrm{~V}$ \\
\hline $\mathrm{C}$ & 0.10 & 0.44 & 0.44 & 0.76 & 0.76 \\
$\mathrm{~V}$ & 0.328 & $<0.005$ & 0.29 & 0.001 & 0.294 \\
$\mathrm{Si}$ & 0.24 & 0.26 & 0.25 & 0.26 & 0.25 \\
$\mathrm{Mn}$ & 0.81 & 0.86 & 0.86 & 0.84 & 0.86 \\
$\mathrm{P}$ & 0.014 & 0.016 & 0.015 & 0.012 & 0.013 \\
$\mathrm{~S}$ & 0.013 & 0.013 & 0.015 & 0.015 & 0.015 \\
$\mathrm{Al}$ & 0.024 & 0.029 & 0.03 & 0.034 & 0.035 \\
$\mathrm{~N}$ & 0.006 & 0.0038 & 0.0045 & 0.0031 & 0.0031 \\
\hline
\end{tabular}




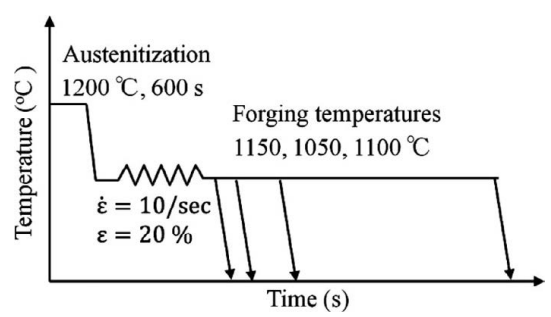

(a)

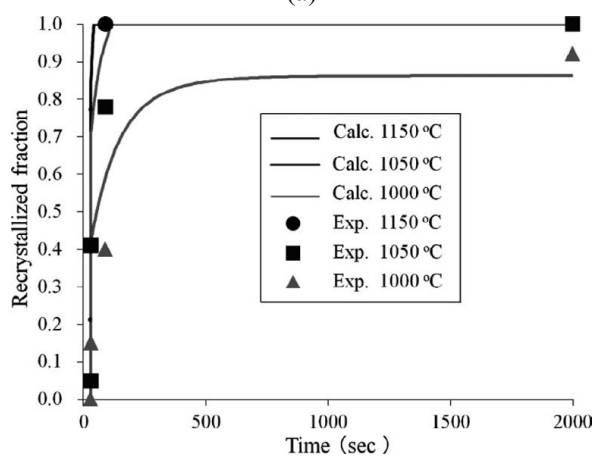

(b)

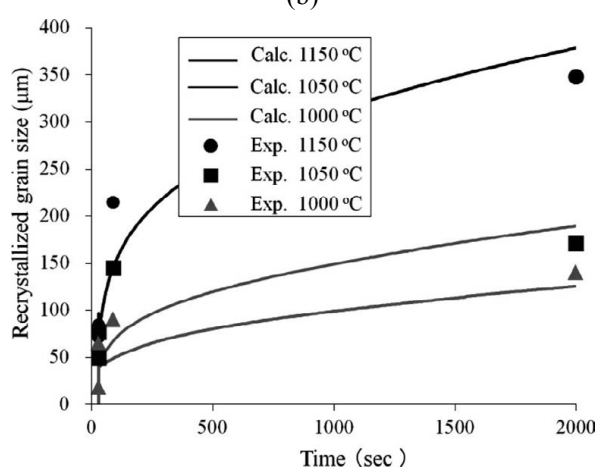

(c)

Fig. 5. (a) Thermo-mechanical pattern showing austenitizing, and forging at different temperatures. The calculated and experimental results of (b) recrystallized fraction and (c) recrystallized grain size are compared.

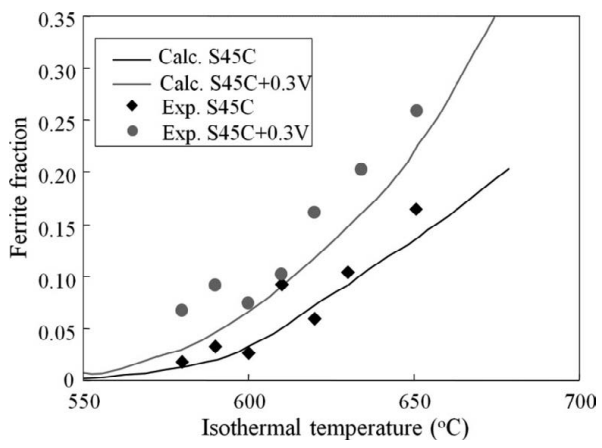

(a)

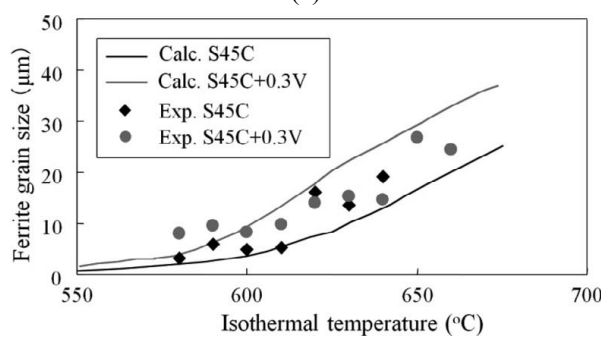

(b)

Fig. 6. Calculated and experimental results of (a) ferrite volume fraction, (b) ferrite grain size in $\mathrm{S} 45 \mathrm{C}$ and $\mathrm{S} 45 \mathrm{C}+0.3 \mathrm{~V}$ after isothermal transformation at different temperatures.
The results in Fig. 5(c) reveal that the VC precipitation during the recrystallization may retard the recrystallized grain growth as well. Based on the same deformation condition, lower deformation temperature will induce higher density of dislocation, from which more VC precipitate. These precipitates will induce the pinning effect on the moving grain boundary, by which the grain growth rate is depressed. Therefore, comparing with the steel deformed at higher temperature, the recrystallized grain size of one deformed at lower temperature is smaller. Again, the volume fraction $\left(f_{v c}\right)$ and radius $\left(r_{v c}\right)$ of $\mathrm{VC}$ are calculated using the model developed by Miyamoto and Furuhara ${ }^{11)}$ to predict the influence of $\mathrm{VC}$ on recrystallization behavior.

The calculated and experimental ferrite fraction and ferrite grain size of $\mathrm{S} 45 \mathrm{C}$ and $\mathrm{S} 45 \mathrm{C}+0.3 \mathrm{~V}$ steels after isothermal transformation at different temperatures are shown in Fig. 6. Since the ferrite fraction of $\mathrm{S} 75 \mathrm{C}$ and $\mathrm{S} 75 \mathrm{C}+0.3 \mathrm{~V}$ is too small at the isothermal temperatures of $550-700^{\circ} \mathrm{C}$, these two steels were regarded as undergone a fully pearlite transformation. Therefore, the experimental ferrite fraction and ferrite grain size of these two steels were not dealt with.

As seen in Fig. 6, the $0.3 \% \mathrm{~V}$ addition into $\mathrm{S} 45 \mathrm{C}$ increases ferrite fraction and grain size. The mechanism is understood that during isothermal transformation, interphase $\mathrm{VC}$ precipitation depletes the carbon at $\alpha / \gamma$ interface, which accelerates carbon diffusivity at interface. In other words, the carbon depletion advances the ferrite formation sufficiently to drag the $\alpha / \gamma$ interface away and VC in sheet is left behind. Meanwhile, VC precipitation also increases the nucleation sites for intragranular ferrite transformation. Since VC effect on ferrite transformation has been taken account, the mathematical model proposed in the present work is valid to predict ferrite transformation.

The calculated and experimental results of lamellar spacing vs. isothermal transformation temperature for $\mathrm{S} 75 \mathrm{C}$ and $\mathrm{S} 75 \mathrm{C}+0.3 \mathrm{~V}$ are shown in Fig. 7. According to the experimental results, the lamellar spacing both in $\mathrm{S} 75 \mathrm{C}$ and $\mathrm{S} 75 \mathrm{C}+0.3 \mathrm{~V}$ decreases with increasing undercooling, and vanadium addition effect on $\mathrm{S} 75 \mathrm{C}$ lamellar spacing is not so obvious. The results show that the present model for lamellar spacing as a function of carbon in austenite and undercooling is valid to predict the lamellar spacing of eutectoid $\mathrm{V}$-free and V-steels.

For medium carbon steels, $\mathrm{S} 45 \mathrm{C}$ and $\mathrm{S} 45 \mathrm{C}+0.3 \mathrm{~V}$, the calculated and experimental lamellar spacing and ferrite volume fraction vs. isothermal transformation temperatures are

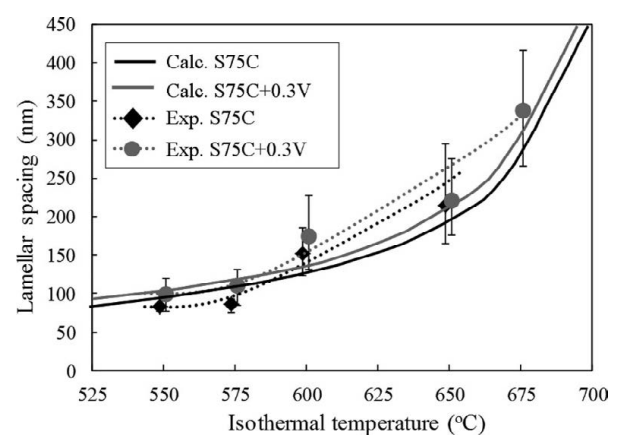

Fig. 7. Calculated and experimental results of lamellar spacing in $\mathrm{S} 75 \mathrm{C}$ and $\mathrm{S} 75 \mathrm{C}+0.3 \mathrm{~V}$ after isothermal transformation at different temperatures. 


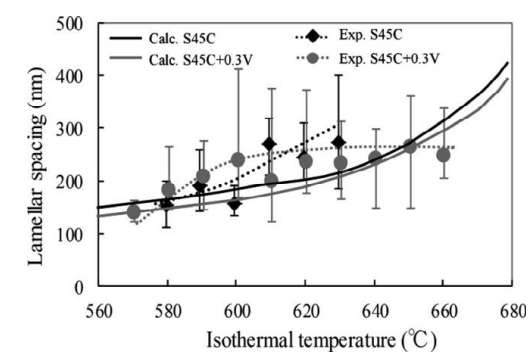

(a)

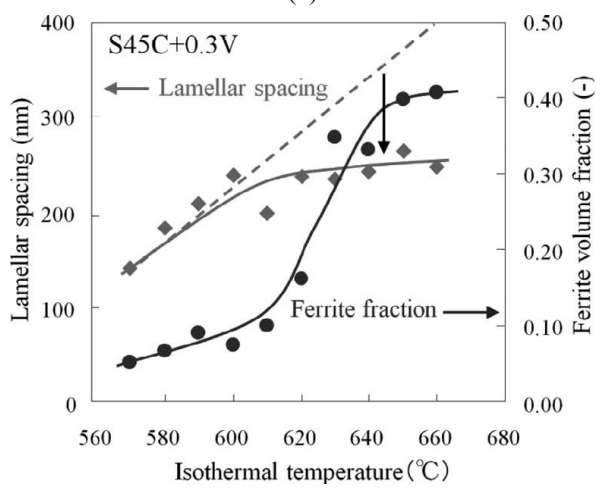

(b)

Fig. 8. (a) Calculated and experimental results of lamellar spacing in $\mathrm{S} 45 \mathrm{C}$ and $\mathrm{S} 45 \mathrm{C}+0.3 \mathrm{~V}$, and (b) the experimental results of lamellar spacing and ferrite volume fraction of $\mathrm{S} 45 \mathrm{C}+0.3 \mathrm{~V}$ after isothermal transformation at different temperatures.

shown in Fig. 8. As seen in Fig. 8.(a), the S45C lamellar spacing decreases with increasing undercooling, that corresponds to the calculated results. However, at temperature range of $600-660^{\circ} \mathrm{C}$ the experimental $\mathrm{S} 45 \mathrm{C}+0.3 \mathrm{~V}$ lamellar spacing almost keeps constant with respect to undercooling.

This phenomenon can be explained by the results shown in Fig. 8(b), which reveals the experimental lamellar spacing and ferrite volume fraction in $\mathrm{S} 45 \mathrm{C}+0.3 \mathrm{~V}$ after isothermal transformation at different temperatures. At 600$660^{\circ} \mathrm{C}$, the ferrite volume fraction is substantially increased mainly due to the increasing allotriomorph and intragranular nucleation density by vanadium addition. Higher ferrite volume fraction results in higher carbon concentration in austenite, and the lamellar spacing is decreased from the value expected by undercooling. Since the effect of increasing ferrite fraction by vanadium addition on lamellar spacing is not taken into account in the present model, it causes the inaccuracy for predicting lamellar spacing of pearlite + ferrite V-steel at some isothermal transformation temperatures. However, this error is not so big for yield strength since the yield strength of V-steel is controlled mainly by interphase VC precipitation strengthening and relatively small by lamellar spacing.

The calculated and experimental yield strengths of $\mathrm{S} 10 \mathrm{C}+0.3 \mathrm{~V}, \mathrm{~S} 45 \mathrm{C}, \mathrm{S} 45 \mathrm{C}+0.3 \mathrm{~V}, \mathrm{~S} 75 \mathrm{C}$, and S75C $+0.3 \mathrm{~V}$ after isothermal transformation at temperature of $550-700^{\circ} \mathrm{C}$ for 600-14 $400 \mathrm{~s}$ are compared in Fig. 9. The accuracy range of the prediction to the yield strength is $\pm 10 \%$. The prediction to yield strength of V-free steels (S45C and S75C) is based on the alloying elements and microstructural factors. As mentioned above, the alloying elements include carbon, silicon, and manganese. Microstructural factors are mainly considered as ferrite and pearlite fractions, ferrite grain size, and lamellar spacing. For the $\mathrm{V}$-steels $(\mathrm{S} 10 \mathrm{C}+0.3 \mathrm{~V}$,

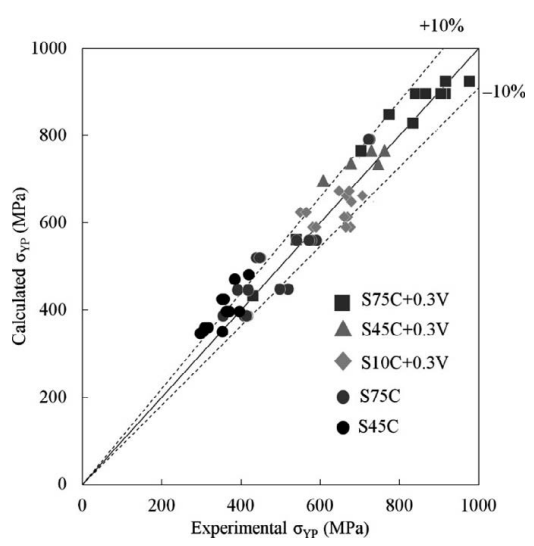

Fig. 9. Calculated and experimental yield strength $\left(\sigma_{Y P}\right)$ of $\mathrm{S} 10 \mathrm{C}+0.3 \mathrm{~V}, \mathrm{~S} 45 \mathrm{C}, \mathrm{S} 45 \mathrm{C}+0.3 \mathrm{~V}, \mathrm{~S} 75 \mathrm{C}, \mathrm{S} 75 \mathrm{C}+0.3 \mathrm{~V}$ steels.

$\mathrm{S} 45 \mathrm{C}+0.3 \mathrm{~V}, \mathrm{~S} 75 \mathrm{C}+0.3 \mathrm{~V})$, the Ashby-Orowan solution is used to calculated $\mathrm{VC}$ precipitation strengthening both in ferrite and pearlite.

According to experimental results of $\mathrm{S} 10 \mathrm{C}+0.3 \mathrm{~V}$ steel with fully ferrite matrix, its yield strength is higher than ones of S45C with ferrite+pearlite and S75C with fully pearlite. It implies that the effect of $\mathrm{VC}$ precipitation on yield strength is stronger than microstructural factors.

As seen from experimental results of $\mathrm{S} 75 \mathrm{C}$, the distribution of yield strength from 400 to $800 \mathrm{MPa}$ is simply due to the variation of lamellar spacing at different isothermal temperatures. However, the $\mathrm{S} 75 \mathrm{C}+0.3 \mathrm{~V}$ steel attains to higher yield strength in the range of $800-1000 \mathrm{MPa}$. The distribution of yield strength in $\mathrm{S} 75 \mathrm{C}+0.3 \mathrm{~V}$ is mainly due to the $\mathrm{VC}$ size and volume fraction rather than lamellar spacing. Similar phenomenon is also found in the case of $\mathrm{S} 45 \mathrm{C}$, whose distribution of yield strength is wider than one of $\mathrm{S} 45 \mathrm{C}+0.3 \mathrm{~V}$ due to the ferrite transformation. Although with higher ferrite fraction and larger ferrite grain size, the yield strength of $\mathrm{S} 45 \mathrm{C}+0.3 \mathrm{~V}$ is more than $200 \mathrm{MPa}$ higher than that of S45C. It is obvious that $\mathrm{VC}$ precipitation in ferrite dominates the strengthening.

\section{Conclusion}

An integrated simulation model for predicting phase transformation and yield strength of vanadium microalloyed carbon steels with carbon concentration of $0.1-0.75$ wt.\% and vanadium of $0-0.3 \mathrm{wt} . \%$ is proposed. The proposed model was compared with experimental data. The main results are concluded as follows:

(1) In the present model, the influence of deformationinduced VC precipitation in austenite on recrystallization behavior has been taken into account. According to the experimental results, the recrystallized fraction and recrystallized grain size decreases with decreasing deformation temperature. It is resulted from the deformation-induced VC precipitation in austenite. At lower deformation temperature, the VC precipitation in austenite occurs during dynamic recrystallization and leads to the pinning effect on grain boundary mobility. With increasing deformation temperature higher than $1150^{\circ} \mathrm{C}$, such precipitation will not occur since $\mathrm{VC}$ is totally solid-solutioned in austenite. By the present model with this consideration, the experimental results are well simulated.

(2) Taking the experimental data into account, the vana- 
dium addition effect on ferrite grain size and ferrite volume fraction are considered in the present model. These effects are (1) changing the para-equilibrium carbon concentration, (2) increasing eutectoid temperature, and (3) interphase VC precipitation. By the model with considering these effects on the increasing of carbon diffusivity in austenite and driving force for ferrite transformation, and enhancement of ferrite growth rate, the calculated results are in good agreement with experimental results. The experimental results showing that ferrite grain size and volume fraction are increased with increasing isothermal temperature and vanadium addition are well simulated.

(3) The present model for predicting lamellar spacing is empirically derived as a function of carbon in austenite and undercooling. The vanadium addition effect on undercooling through increasing the eutectoid temperature is considered. This model is valid to predict the lamellar spacing of pearlite $\mathrm{V}$ - and V-free steels since the experimental results show that the lamellar spacing of pearlite steel depends on undercooling rather than vanadium content. However, for pearlite+ferrite $\mathrm{V}$-steels, the vanadium addition increases the ferrite volume fraction, which leads to austenite having higher carbon content and the lamellar spacing is therefore decreased. Since the yield strength of V-steel is controlled mainly by interphase VC precipitation strengthening and relatively small by lamellar spacing, the inaccuracy between experimental and calculated lamellar spacing for V-steel is acceptable to predict the yield strength.

(4) Assuming VC precipitate is an unshearable particle, the Ashby-Orowan theory is therefore conducted to develop the equation for $\mathrm{VC}$ precipitation strengthening in $\mathrm{V}$-steels. This equation is a function of $\mathrm{VC}$ size and volume fraction. According to the results, the precision to the prediction of yield strength is in $\pm 10 \%$.

\section{Acknowledgements}

Part of this work was carried out as a part of "Fundamental Studies on Technologies for Steel Materials with Enhanced Strength and Functions" by Consortium of JRCM. Financial support from NEDO is gratefully acknowledged.

\section{REFERENCE}

1) Y. Estrin: in Unified Constitutive Laws of Plastic Deformation, ed. by A. S. Krausz and K. Krausz. Academic Press, London, (1996), 69.

2) Y. C. Lin, M. S. Chen and J. Zhong: J. Mater. Process. Tech., 209 (2009), 2477.

3) C. A. C. Imbert and H. J. McQueen: Mater. Sci. Eng. A, 313 (2001), 104.

4) I. Salvatori, T. Inoue and K. Nagai: ISIJ Int., 42 (2002), 744.

5) P. L. Mao, G. Y. Su and K. Yang: Mater. Sci. Technol., 18 (2002), 892.

6) P. Peczak and M. J. Luton: Philos. Mag. B, 68 (1993), 115.

7) P. Peczak and M. J. Luton: Philos. Mag. B, 70 (1994), 817.

8) P. Peczak: Acta Metall. Mater., 43 (1995), 1279.

9) R. Ding and Z. X. Guo: Acta Mater., 49 (2001), 3163.

10) R. Kuziak, Z. Malinowski and M. Pietrzyk: Proc. of AMT'98 on Advanced Materials and Technologies, AMT98', Krynica, Poland, (1998), 685.

11) P. A. Manohar, D. P. Dunnet, T. Chandra and C. R. Killmore: ISIJ Int., 36 (1996), 194.

12) G. Miyamoto and T. Furuhara: 160th ISIJ Proc., ISIJ, Tokyo, (2010).

13) J. W. Cahn: Acta Metall., 10 (1962), 789.

14) H. S. Zurob, Y. Brechet and G. Purdy: Acta Mater., 49 (2001), 4183.

15) C. S. Simth: Trans. Am. Inst. Min. Eng., 175 (1948), 15.

16) H. Yada: JSTP, 28 (1987), 413.

17) A. Kumar: Steel Res., 64 (1993), 210.

18) H. I. Aaronson: Symp. on the Mechanical Properties of Phase Transformations in Metals, Institute of Materials, London, (1995), 47.

19) W. F. Lange, M. Enomoto and H. I. Aaronson: Metall. Trans. A, 19 (1988), 427.

20) J. R. Bradley, J. M. Rigsbee and H. I. Aaronson: Metall. Trans. A, 8 (1977), 323.

21) L. Kaufman, S. V. Radcliffe and M. Cohen: Decomposition of Austenite by Diffusional Processes, Interscience Publishers, New York, (1962), 313.

22) J. W. Christian: The Theory of Transformation in Metals and Alloys, 2nd Edition, Pergamon Press, Oxford, (1975).

23) R. F. Mehl: Hardenability of Alloy Steels, Am. Soc. for Metals Symp., ASM, Ohio, USA, (1939).

24) R. F. Mehl: 16th Campbell Memorial Lecture, Am. Soc. for Metals, ASM, Ohio, USA, (1941).

25) W. A. Johnson and R. F. Mehl: Trans. AIME, 135 (1939), 416

26) F. C. Hull and R. F. Mehl: Trans. Am. Soc. Met., 39 (1942), 381

27) F. C. Hull, R. A. Colton and R. F. Mehl: Trans. AIME., 150 (1942), 185.

28) W. H. Brandt: Some Factors Affecting Edgewise Growth of Pearlite, New York Meeting, Metals Technology, New York, (1945), 405.

29) T. Gladman, I. D. Mcivor and F. B. Pickering: J. Iron Steel Inst., 12 (1972), 916

30) F. B. Pickering and T. Gladman: Metallurgical developments in carbon steels, The Iron and Steel Institute, London, (1963). 10.

31) M. Gensamer: IBID, 30 (1942), 983.

32) E. Orowan: Internal stress in metals and alloy, The Institute of Metals, London, (1948), 451.

33) M. F. Ashby: Oxide dispersion strengthening, ed. by G. S. Ansell et al., Gordon and Breach, New York, (1958), 143. 